\title{
Radionuclide Imaging of Atherothrombotic Diseases
}

\author{
Mitchel R. Stacy ${ }^{1,2}$ \\ Published online: 27 March 2019 \\ (C) The Author(s) 2019
}

\begin{abstract}
Purpose of Review A variety of approaches and molecular targets have emerged in recent years for radionuclide-based imaging of atherosclerosis and vulnerable plaque using single photon emission computed tomography (SPECT) and positron emission tomography (PET), with numerous methods focused on characterizing the mechanisms underlying plaque progression and rupture. This review highlights the ongoing developments in both the pre-clinical and clinical environment for radionuclide imaging of atherosclerosis and atherothrombosis.

Recent Findings Numerous physiological processes responsible for the evolution of high-risk atherosclerotic plaque, such as inflammation, thrombosis, angiogenesis, and microcalcification, have been shown to be feasible targets for SPECT and PET imaging. For each physiological process, specific molecular markers have been identified that allow for sensitive non-invasive detection and characterization of atherosclerotic plaque.

Summary The capabilities of SPECT and PET imaging continue to evolve for physiological evaluation of atherosclerosis. This review summarizes the latest developments related to radionuclide imaging of atherothrombotic diseases.
\end{abstract}

Keywords Atherosclerosis $\cdot$ Atherothrombosis $\cdot$ Molecular imaging $\cdot$ SPECT $\cdot$ PET $\cdot$ Radionuclide imaging

\section{Introduction}

Cardiovascular disease remains a leading cause of death worldwide, with many adverse cardiovascular events and deaths being attributable to acute myocardial infarction due to coronary vessel atherosclerosis and/or thrombosis and atherothrombosis-related ischemic stroke [1]. The underlying atherosclerosis responsible for the majority of adverse cardiovascular events is a complex disease that is characterized by formation and progression of occlusive vascular plaques that arise due to a variety of factors, which include, but are not limited to, inflammatory mediators, endothelial injury, infiltration of oxidized low-density lipoproteins, calcium deposition, and plaque neoangiogenesis $[2,3]$. While the atherosclerotic process can lay dormant for months, years, or decades,

Mitchel R. Stacy

mitchel.stacy@nationwidechildrens.org

1 Department of Surgery, The Ohio State University College of Medicine, 575 Children's Crossroad, WB4131, Columbus, $\mathrm{OH} 43215$, USA

2 The Center for Regenerative Medicine, The Research Institute at Nationwide Children's Hospital, Columbus, OH, USA erosion or damage to the fibrous cap of an atherosclerotic plaque can have severe health consequences, such as the triggering of occlusive thrombosis and subsequent development of tissue ischemia [4]. Atherothrombosis can be initiated through the exposure of thrombogenic components contained within the plaque following fissure or erosion of the plaque's surface, which ultimately facilitates platelet aggregation on the exposed plaque surface and results in subsequent fibrin and thrombus formation $[4,5]$. In general, stable plaques typically possess small necrotic cores and thick fibrous caps, while rupture prone vulnerable plaques that are susceptible to triggering atherothrombotic events are usually characterized by larger necrotic cores and thinner fibrous caps [6]. Histologically, rupture- and thrombotic-prone plaques are also commonly characterized by extensive macrophage infiltration, neovascularization, remodeling of the extracellular matrix, apoptosis, and smooth muscle cell proliferation $[3,7]$.

The non-invasive diagnosis of thrombotic diseases and vulnerable plaque has traditionally been limited to indirect detection through the discovery of associated blood flow or tissue perfusion deficits. However, considering the various thrombispecific epitopes (e.g., fibrin, epitopes on activated platelets, activated coagulation factors) that exist as potential targets for molecular imaging, along with the ongoing developments in 
modern day imaging technologies that now allow for both high-resolution anatomical and high-sensitivity molecular imaging, a number of non-invasive approaches are emerging that are offering hope for improving the detection of vulnerable plaque and assisting with stratification of at-risk patients. Specifically, intravascular imaging modalities such as optical coherence tomography (OCT) and intravascular ultrasound (IVUS) now provide high-resolution imaging of plaque characteristics in patients undergoing catheterization [8], while more traditional techniques such as X-ray computed tomography $(\mathrm{CT})$ and magnetic resonance (MR) imaging can offer non-invasive morphological insight into plaque calcium burden [9] and the presence of plaque hemorrhage [10], respectively.

\section{Radionuclide Imaging}

The radionuclide-based imaging modalities of single photon emission computed tomography (SPECT) and positron emission tomography (PET) have also begun to play a role in the non-invasive evaluation of various molecular processes associated with thrombosis and vulnerable plaque. SPECT and PET are capable of producing three-dimensional (3D) physiological images via detection of gamma rays that are emitted from injected radiopharmaceuticals, with these targeted radiolabeled probes of variable half-lives and energies being utilized for numerous strategies depending on the ultimate goal of the imaging exam. Both SPECT and PET possess superior sensitivity for targeted molecular imaging when compared to other commercially available imaging modalities; however, conventional SPECT and PET scanners also possess relatively poor spatial resolution (SPECT $5-8 \mathrm{~mm}^{3}$; PET 3$5 \mathrm{~mm}^{3}$ ). Therefore, the majority of modern SPECT and PET scanners now incorporate and are paired with high spatial resolution $\mathrm{CT}$ or MR scanners as part of hybrid systems (i.e., SPECT/CT, PET/CT, PET/MR), which offer the benefits of co-localizing high sensitivity functional imaging and highresolution anatomical imaging [11].

When comparing the radionuclides available for SPECT and PET imaging, SPECT radionuclides are generally widely available, involve rather simple radiochemistry, and commonly possess relatively long half-lives. Alternatively, PET radionuclides possess characteristics that generally include shorter half-lives and more complex production/radiochemistry, both qualities that limit the widespread availability of certain PET tracers. With regard to modality comparisons, PET provides numerous advantages over SPECT. Specifically, PET possesses superior sensitivity and spatial resolution when compared to SPECT, and the recent emergence of digital PET scanners is now resulting in further improvements in image resolution down to ultra-high definition at $1 \times 1 \times 1 \mathrm{~mm}^{3}$ voxel sizes [12]. Additionally, PET has established methodology for absolute quantification of radionuclide uptake, while SPECT has traditionally focused on the evaluation of relative radionuclide retention. While PET does possess advantages, SPECT has been and remains a mainstay in the clinical environment for evaluation of myocardial perfusion due to its lower relative cost and wider availability and offers potential opportunities for dual isotope targeted molecular imaging that is not always feasible with PET imaging. Additionally, the recent emergence of cadmium zinc telluride solid-state detectors for SPECT continues to enhance and expand the translational capabilities of SPECT imaging [13].

The ultimate quantitative assessment of radionuclide imaging in the vasculature remains a topic of debate, but various methods have been evaluated and established for the purposes of SPECT and PET image analyses. Specifically, standardized uptake values (SUVs) and target-to-background ratios (TBRs) have been the traditional methods utilized when quantifying retention of targeted radionuclides in the vasculature. SUVs are calculated based on the radionuclide retention within the region of interest after correcting for the injected radionuclide dose and the patient's body weight. SUVs can then be further analyzed and expressed based on the maximum $\left(\mathrm{SUV}_{\max }\right)$ and/or mean $\left(\mathrm{SUV}_{\text {mean }}\right)$ count statistics within the vascular region of interest. Alternatively, TBR values, which are commonly used in the analysis of atherosclerotic plaques identified on PET/CT images, are calculated based on acquiring SUVs in both the vessel wall of interest as well as the vessel lumen, so that the vessel wall serves as the "target" and the blood pool serves as the "background" [14].

Regardless of the image analysis techniques utilized, a variety of radionuclides exist and continue to emerge for SPECT- and PET-based molecular imaging of atherothrombosis and vulnerable plaque that are capable of targeting physiological processes such as inflammation, thrombosis, calcium deposition, and angiogenesis [15]. The following sections of this review highlight the recent advancements related to SPECT and PET imaging of atherosclerosis, atherothrombosis, and vulnerable plaque, with particular focus on emerging radionuclides and physiological targets for non-invasive molecular imaging.

\section{Radionuclide Imaging of Vascular Inflammation}

A physiological process that plays a major role in regulating plaque vulnerability is vascular inflammation. In general, atherosclerosis is promoted by inflammatory responses that occur following the infiltration, accumulation, and oxidation of lowdensity lipoproteins (LDL) in the vascular wall. Following LDL oxidation, inflammatory mediators such as Tlymphocytes and macrophages respond to consume LDL and ultimately form foam cells. While this inflammatory 
response is designed to be protective in nature, macrophage accumulation in the lesion site can ultimately trigger further inflammation and the subsequent creation of a thin fibrous capped atheroma that is susceptible to rupture [10].

Due to the key role that macrophages play in atherosclerotic plaque evolution and vulnerability for rupture, PET imaging of fluorine-18 $\left({ }^{18} \mathrm{~F}\right)$-fluorodeoxyglucose $(\mathrm{FDG})$, a radiolabeled glucose analog that is retained by metabolically active macrophages, has been one of the most widely used radionuclide-based approaches for assessing atherosclerosis. Clinical research in the last 5 years has demonstrated in a variety of studies that ${ }^{18} \mathrm{~F}-\mathrm{FDG}$ PET/CT imaging possesses clinical utility for non-invasively detecting vascular inflammation in the setting of atherosclerosis [16••, 17-21]. Recent clinical studies have specifically shown that ${ }^{18} \mathrm{~F}$-FDG uptake in the ascending aorta possesses prognostic for predicting future cardiovascular events [20] and is associated with high rates of coronary heart disease events [22]. Additionally, ${ }^{18} \mathrm{~F}$ FDG PET/CT imaging has demonstrated the ability to detect the effectiveness of various statin dosing regimens at reducing plaque inflammation $[16 \bullet \bullet, 19]$. While ${ }^{18}$ F-FDG PET/CT imaging has demonstrated utility for predicting cardiovascular events and tracking therapy in patients, vascular ${ }^{18} \mathrm{~F}$-FDG uptake has also been shown to be significantly elevated in patients with elevated lipoprotein(a) levels [23] and significantly correlate with circulating levels of myeloperoxidase [18] and total plasma cholesterol [21]. Recent work by Cocker et al. [24] further revealed that ${ }^{18} \mathrm{~F}-\mathrm{FDG}$ uptake specifically represents a non-invasive surrogate marker of macrophage and leukocyte infiltration by demonstrating that ${ }^{18} \mathrm{~F}$ FDG uptake in carotid artery endarterectomy specimens significantly correlated with both CD68 macrophage expression and CD45 leukocyte expression. Along with prior studies that have utilized ${ }^{18} \mathrm{~F}$-FDG, additional work by Tahara and colleagues [25] has applied a similar macrophage targeted approach by utilizing ${ }^{18} \mathrm{~F}$-labeled mannose (2-deoxy$2-\left[{ }^{18} \mathrm{~F}\right]$ fluoro-D-mannose; $\left.{ }^{18} \mathrm{~F}-\mathrm{FDM}\right)$, an isomer of glucose, which revealed similar uptake patterns to ${ }^{18} \mathrm{~F}-\mathrm{FDG}$ in the evaluation of atherosclerotic plaques and also showed uptake patterns that were proportional to plaque macrophage infiltration. Recently, various other approaches, such as gallium-68 $\left({ }^{68} \mathrm{Ga}\right)$-DOTATATE, a somatostatin receptor subtype-2 targeted PET probe [26 ${ }^{\bullet}$, and zirconium-89 $\left({ }^{89} \mathrm{Zr}\right.$ )-labeled dextran nanoparticles [27], have demonstrated potential for molecular imaging of plaque inflammation and have shown uptake patterns consistent with the extent of macrophage infiltration, thus revealing that various radionuclide techniques may ultimately be feasible and clinically available for targeted imaging of macrophage-mediated plaque inflammation.

Although PET-based assessment of macrophage activity has composed the majority of inflammation targeted imaging investigations, other techniques have emerged in recent years that have focused on various other mediators of inflammation.
For example, molecules such as P-selectin and vascular cell adhesion molecule-1 (VCAM-1), which play important roles in the recruitment and adhesion of key inflammatory cells, have served as imaging targets for investigating plaque inflammation in pre-clinical models of atherosclerosis [28-30]. Specifically, Li et al. [28] utilized ${ }^{68}$ Ga-Fucoidan for PET/MR imaging of P-selectin expression in apolipoprotein E-deficient mice and demonstrated increased radionuclide uptake in plaque with thin fibrous caps and a high density of foam cells, while other research teams have utilized both SPECT- [29] and PET-based [30] approaches to target VCAM-1 and have had success using similar mouse and rabbit models of atherosclerosis. In addition to imaging of P-selectin and VCAM-1, less studied techniques have also included the use of SPECT/ CT imaging of technetium-99 $\mathrm{m}\left({ }^{99 \mathrm{~m}} \mathrm{Tc}\right)$-labeled peripheral blood mononuclear cells for evaluating the consequences of circulating monocytes on plaque inflammation [23], as well as ${ }^{68}$ Ga-pentixafor PET/CT imaging for assessing CXC-motif chemokine receptor 4 (CXCR4), with the latter approach demonstrating a significant association with various cardiovascular risk factors and showing increased radionuclide uptake in regions of calcified atherosclerotic lesions (Fig. 1) [31].

\section{Radionuclide Imaging of Thrombosis}

Along with targeted imaging of plaque inflammation, recent research has also focused on non-invasive imaging of various molecular markers associated with thrombosis. The end product of the coagulation cascade, fibrin, has served as one of the most widely investigated imaging markers of thrombus formation, with fibrin being non-invasively detected in pre-clinical models of thrombosis using radiolabeled probes targeting various fibrin binding peptides (FBPs). For example, Ciesienski and colleagues [32] conjugated three different FBPs (FBP1, FBP2, FBP3) as 1,4,7,10-tetraazacyclododecane$1,4,7,10$,tetraacetic acid (DOTA)-monoamides for labeling with copper-64 $\left({ }^{64} \mathrm{Cu}\right)$ and demonstrated with hybrid PET/MR imaging that uptake of FBP1 and FBP2 probes was elevated approximately fourfold above control levels when evaluating a rat model of carotid artery thrombosis. Additional work by Ay et al. [33*0] utilized a ${ }^{64} \mathrm{Cu}$-labeled $\mathrm{FB} 7$ probe for the in vivo evaluation of a rat model of embolic stroke that underwent thrombolysis with recombinant tissue-type plasminogen activator (rtPA), which revealed the ability of ${ }^{64} \mathrm{Cu}-\mathrm{FB} 7 \mathrm{PET} / \mathrm{CT}$ imaging to non-invasively detect response to treatment. Similarly, Blasi and colleagues [34] utilized a ${ }^{64} \mathrm{Cu}$-labeled FBP8 probe for PET/CT imaging and revealed the ability of ${ }^{64} \mathrm{Cu}$-FBP8 to detect blood clots of varying ages (i.e., 1, 3, or 7 days after induction of thrombosis) in a rat model, with imaging findings confirmed by both histology and autoradiography. Although ${ }^{64} \mathrm{Cu}$-labeled FBPs have proven to be effective for PET imaging of fibrin, recent work by Oliveira et al. [32] 
has also demonstrated that FBP targeted probes such as ${ }^{68} \mathrm{Ga}-$ labeled FBP14 and indium-111 ( ${ }^{111}$ In)-FBP15 possesses high affinity for fibrin and good specificity for thrombus detection, thereby representing potential radionuclides that could be utilized for PET- or SPECT-based imaging of thrombus formation.

While fibrin-targeted imaging has represented the majority of thrombosis focused investigations, factor XIII (FXIII), an enzyme involved in the coagulation cascade and crosslinking of fibrin, has also served as a molecular probe for thrombosis research. Specifically, Chen and colleagues [35] demonstrated that an ${ }^{111}$ In-labeled peptide targeting FXIII was capable of detecting elevated activation of the coagulation cascade in a mouse model of silent brain ischemia by using SPECT/CT imaging and showed further utility of ${ }^{111}$ In-FXIII SPECT/ CT imaging through the non-invasive tracking of therapyinduced reductions in FXIII activity. Additionally, recent work by Zhuang et al. [36] has utilized a ${ }^{99 \mathrm{~m}}$ Tc-labeled FXIII targeted probe for SPECT/CT imaging and shown sensitivity for detecting the formation of cross-linked fibrin in the microcirculation of the mouse heart.

In addition to fibrin and FXIII imaging, SPECT and PET research has also been conducted in the pre-clinical setting to non-invasively evaluate activated platelets as potential biomarkers of atherothrombosis. In an attempt to image activated platelets, previous research in mouse models of carotid artery thrombosis has focused on radionuclides that selectively targeted the ligand induced binding sites (LIBS) epitope of the receptor site for glycoprotein IIb/IIIa that resides on the surface of platelets [37, 38]. Specific work by Heidt and colleagues [39] have demonstrated that ${ }^{111}$ In-labeled LIBS specifically binds to activated platelets and can be utilized for SPECT/CT imaging of carotid artery thrombosis, while Ardipradja and colleagues [40] have more recently shown the feasibility of utilizing an ${ }^{18} \mathrm{~F}$-labeled LIBS probe, ${ }^{18} \mathrm{~F}$ $\mathrm{scFv}_{\text {anti-LIBS }}$, for PET/CT imaging of carotid artery thrombosis in a mouse model.

\section{Radionuclide Imaging of Plaque Angiogenesis}

Angiogenesis, which is initiated and promoted when plaques develop thick necrotic and hypoxic cores, remains another important physiological process that can contribute to both plaque growth and vulnerability to rupture [41]. Therefore, molecular targets, such as the $\alpha \mathrm{v} \beta 3$ integrin and vascular endothelial growth factor (VEGF), have emerged in recent years for SPECT- and PET-based molecular imaging of angiogenesis in high-risk plaques [39, 40, 42, 43]. For example, Yoo and colleagues [39॰] developed the $\alpha v \beta 3$ integrin targeted probe, ${ }^{99 \mathrm{~m}} \mathrm{Tc}-\mathrm{IDA}-\mathrm{D}-[\mathrm{c} \text { (RGDfk) }]_{2}$, for the application of SPECT/CT imaging in a mouse model of atherosclerosis and demonstrated significant elevations in radionuclide uptake within atherosclerotic aortas, with confirmatory histological analysis that revealed probe uptake co-localized to plaques possessing large necrotic cores and thin fibrous caps. Additional pre-clinical work by Jiang and colleagues [40]


Fig. $1{ }^{68} \mathrm{Ga}$-pentixafor PET/CT imaging of abdominal aorta inflammation. Transaxial views of $\mathbf{a} \mathrm{CT}$ and $\mathbf{b}$ fused PET/CT images reveal localization of ${ }^{68} \mathrm{Ga}$-pentixafor uptake to regions of partial vascular calcification (denoted by white arrows). Sagittal views of $\mathbf{c}$ CT and $\mathbf{d}$ fused PET/CT images demonstrate ${ }^{68} \mathrm{Ga}$-pentixafor uptake in partially calcified and non-calcified atherosclerotic lesions (dashed arrows). (This research was originally published in JNM. Weiberg, Thackeray, Daum, et al. Clinical Molecular Imaging of Chemokine Receptor CXCR4 Expression in Atherosclerotic Plaque Using ${ }^{68} \mathrm{Ga}-$ Pentixafor PET: Correlation with Cardiovascular Risk Factors and Calcified Plaque Burden. J Nucl Med. 2018;59:266-272. @SNMMI.) 
Fig. $2{ }^{64} \mathrm{Cu}-\mathrm{NOTA}-3-4 \mathrm{~A}$ PET/CT imaging for detection of integrin expression in a mouse model of carotid artery atherosclerosis. a PET, CT, and fused PET/CT imaging $1 \mathrm{~h}$ after injection of ${ }^{64} \mathrm{Cu}$ NOTA-3-4A revealed radionuclide uptake in the site of the carotid artery atherosclerotic lesion. $\mathbf{b}$ Coronal PET images of carotid artery plaques at $1,2,4$, and $24 \mathrm{~h}$ after injection of ${ }^{64} \mathrm{Cu}$-NOTA-3$4 \mathrm{~A}$ without or with c (RGDyK), a blocking agent, which demonstrated suppression of ${ }^{64} \mathrm{Cu}$ NOTA-3-4A uptake. c Quantitative analysis of radionuclide retention in organs at various time points following injection of ${ }^{64} \mathrm{Cu}-$ NOTA-3-4A, expressed as \%ID/g. d Quantitative analysis of plaqueto-normal tissue ratios at various time points. \% ID/g percent injected dose per gram, B blood, $\mathrm{H}$ heart, $\mathrm{K}$ kidney, Li liver, $\mathrm{M}$ muscle, $\mathrm{N}$ normal vessel wall, $\mathrm{P}$ plaque. (This research was originally published in JNM. Jiang, Tu, Kimura, et al.

${ }^{64} \mathrm{Cu}$-Labeled Divalent Cystine Knot Peptide for Imaging Carotid Atherosclerotic Plaques. J Nucl Med. 2015;56:939-944. (CSNMMI.) a
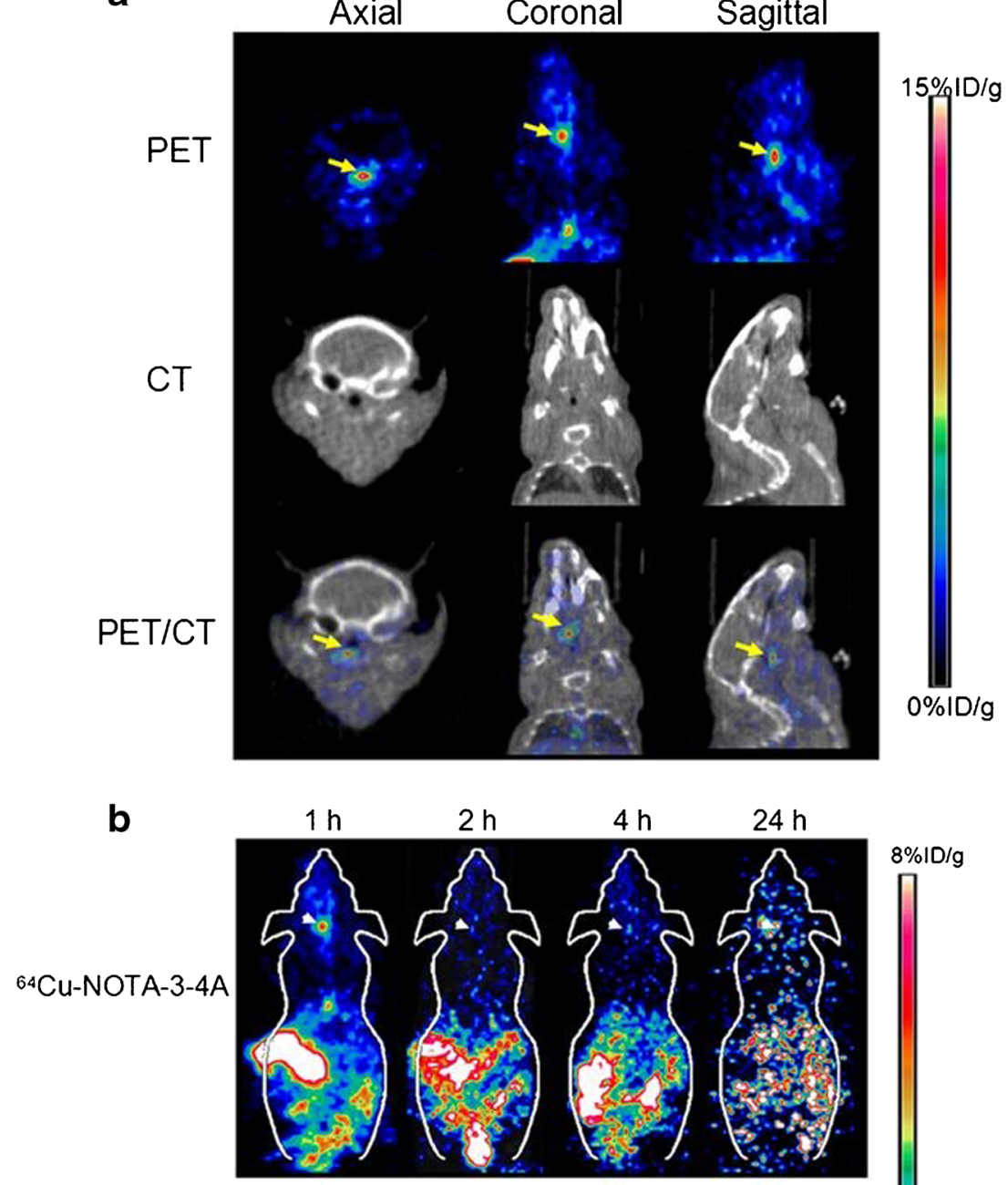

$8 \% 10 / g$

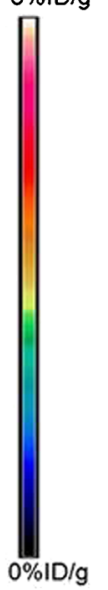

C



d

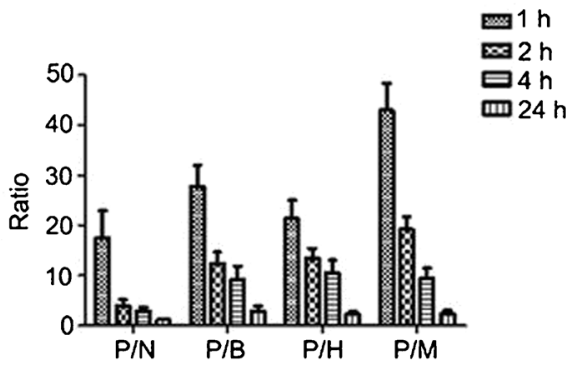


utilized a PET probe, ${ }^{64} \mathrm{Cu}$-NOTA-3-4A, in a mouse model of atherosclerosis to image $\alpha \mathrm{v} \beta 3$ integrin expression and showed significant elevations in radionuclide uptake within atherosclerotic lesions of the carotid arteries, as well as specificity of the tracer for targeting plaque (Fig. 2). Along with research in pre-clinical models of atherosclerosis, Golestanie et al. [42] have also evaluated the potential clinical translatability of a ${ }^{18} \mathrm{~F}$-RGD probe for assessing $\alpha \mathrm{v} \beta 3$ integrin expression by incubating human carotid endarterectomy specimens in the radionuclide and then applying microPET imaging, which demonstrated significant correlations between radionuclide uptake and integrin staining score, as well as radionuclide uptake and CD31 staining score. While various radionuclides have proven to be effective for imaging of $\alpha v \beta 3$ integrin expression, more recent work by Tekabe et al. [43] has applied a ${ }^{99 \mathrm{~m}} \mathrm{Tc}-$ labeled probe for SPECT/CT targeted imaging of VEGF receptor-1 (VEGFR-1) and VEGF receptor-2 (VEGFR-2) in apolipoprotein E deficient mice and shown significant increases specifically in VEGFR-1 signaling within atherosclerotic aortas and brachiocephalic vessels, thus revealing a novel target for imaging of intraplaque angiogenesis that may assist in guiding future research in this field.

\section{Radionuclide Imaging of Plaque Microcalcification}

Generally regarded as a natural healing response to extensive inflammation within plaque, calcification of atherosclerotic lesions has been traditionally characterized as late-stage macrocalcification, where plaque has reached a phase of stabilization, or early-stage microcalcification, where plaque remains in an unstable and vulnerable state due to disruption of the fibrous cap and/or ongoing inflammation in the region of the plaque surrounding the calcium deposits [10]. The radionuclide primarily utilized for investigating active microcalcification in atherosclerotic plaques has been ${ }^{18} \mathrm{~F}$-sodium fluoride $(\mathrm{NaF})$ due to its high affinity and specificity for calcified deposits [44••]. The mechanism of uptake for ${ }^{18} \mathrm{~F}-\mathrm{NaF}$ is based on $\mathrm{NaF}$ replacing the hydroxyl groups of hydroxyapatite, which has been shown to be present in areas of active microcalcification [45]. Early research with ${ }^{18} \mathrm{~F}-\mathrm{NaF}$ PET/CT imaging in the setting of carotid artery atherosclerosis demonstrated that ${ }^{18} \mathrm{~F}-\mathrm{NaF}$ uptake co-localized with regions of arterial calcification (Fig. 3), was more prevalent in patients at high risk for atherothrombotic events, and significantly correlated with the presence of multiple cardiovascular risk factors, such as diabetes, history of smoking, and prior history of cardiovascular events [46॰]. Additional work by other research teams has similarly revealed that patients with high coronary artery ${ }^{18} \mathrm{~F}$ $\mathrm{NaF}$ uptake also had a higher prevalence of arterial calcification and history of myocardial infarction or unstable angina [47], as well as higher rates of prior cardiovascular events and higher Framingham risk scores [48]. While in vivo clinical imaging studies have shown a close link between ${ }^{18} \mathrm{~F}-\mathrm{NaF}$ uptake and cardiovascular history/risk, more recent research has also focused on histological validation of ${ }^{18} \mathrm{~F}-\mathrm{NaF}$ uptake to better understand the mechanisms associated with ${ }^{18} \mathrm{~F}-\mathrm{NaF}$ retention in high-risk plaques. Specifically, recent work by Joshi et al. [49] has demonstrated that ${ }^{18} \mathrm{~F}-\mathrm{NaF}$ uptake in carotid artery endarterectomy specimens is associated with histological evidence of active calcification, macrophage infiltration, apoptosis, and necrosis, while additional research by Irkle et al. [44••] has shown that ${ }^{18} \mathrm{~F}-\mathrm{NaF}$ can differentiate between regions of macro- and microcalcification in atherosclerotic plaque. Furthermore, Cocker and colleagues [50] have recently revealed in excised human carotid artery plaques that ${ }^{18} \mathrm{~F}-\mathrm{NaF}$ uptake significantly correlates with Goldner's trichrome staining, a method for histologically detecting the presence of hydroxyapatite, thus demonstrating that ${ }^{18} \mathrm{~F}-\mathrm{NaF}$ is related to the process of active microcalcification in plaque and may serve as a useful non-invasive biomarker for characterizing high-risk plaque that is vulnerable to rupture.
Fig. 3 In vivo ${ }^{18} \mathrm{~F}-\mathrm{NaF}$ PET/CT imaging of the common carotid arteries. Transaxial views of a CT, b ${ }^{18} \mathrm{~F}-\mathrm{NaF}$ PET, and $\mathbf{c}$ fused PET/ CT images demonstrate bilateral uptake of ${ }^{18} \mathrm{~F}-\mathrm{NaF}$ in common carotid artery plaques that is localized to sites of vascular calcification. (This research was originally published in JNM. Derlin, Wisotzki, Richter, et al. In Vivo Imaging of Mineral Deposition in Carotid Plaque Using ${ }^{18}$ F-Sodium Fluoride PET/ CT: Correlation with Atherogenic Risk Factors. J Nucl Med. 2011;52:362-368. OSNMMI.)
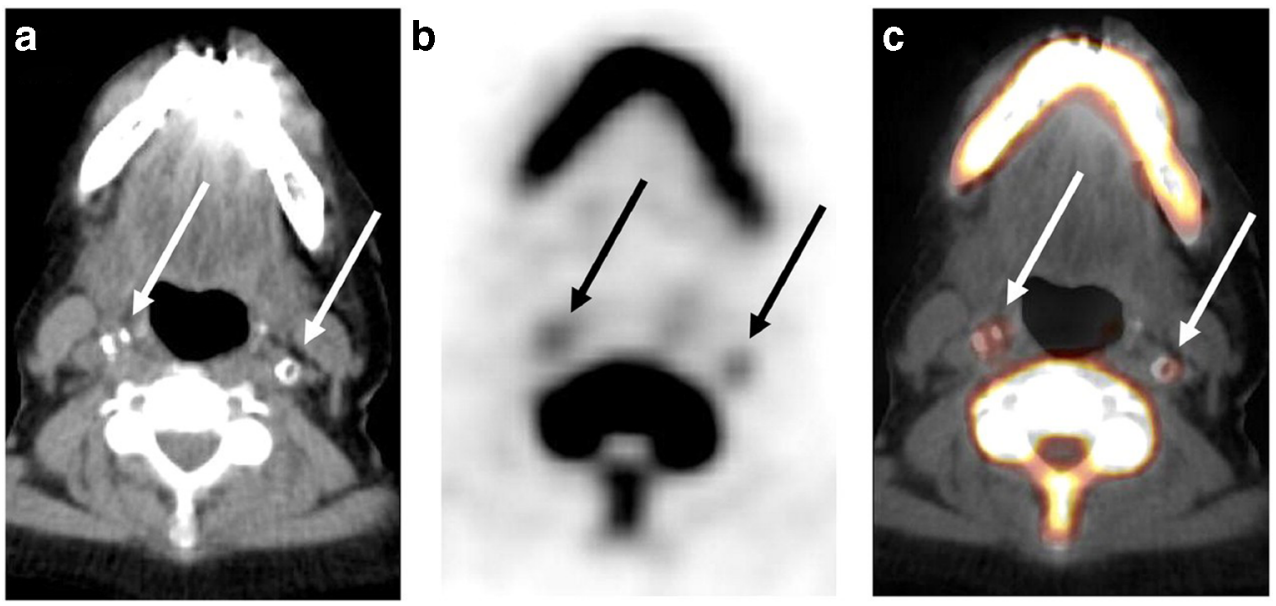
Table 1 Radionuclides for non-invasive imaging of atherothrombosis and plaque vulnerability

\begin{tabular}{|c|c|c|c|}
\hline Physiologic target & Radionuclide & Imaging modality & Reference \\
\hline \multirow[t]{9}{*}{ Inflammation } & ${ }^{68} \mathrm{Ga}$-DOTATATE & $\mathrm{PET} / \mathrm{CT}$ & {$[26 \bullet]$} \\
\hline & ${ }^{68}$ Ga-fucoidan & PET/MR & {$[28]$} \\
\hline & ${ }^{68} \mathrm{Ga}$-pentixafor & $\mathrm{PET} / \mathrm{CT}$ & [31] \\
\hline & ${ }^{99 \mathrm{~m}} \mathrm{Tc}$-mononuclear cells & $\mathrm{SPECT} / \mathrm{CT}$ & {$[23]$} \\
\hline & ${ }^{99 \mathrm{~m}} \mathrm{Tc}-\mathrm{scFv}-\mathrm{VCAM} 1$ & $\mathrm{SPECT} / \mathrm{CT}$ & {$[29]$} \\
\hline & ${ }^{18} \mathrm{~F}-\mathrm{FB}$-anti-VCAM-1 nanobody & $\mathrm{PET} / \mathrm{CT}$ & {$[30]$} \\
\hline & ${ }^{89} \mathrm{Zr}$-dextran nanoparticle & PET/MR & {$[27]$} \\
\hline & ${ }^{18} \mathrm{~F}-\mathrm{FDG}$ & $\mathrm{PET} / \mathrm{CT}$ & {$[16 \bullet \bullet, 17-22,24]$} \\
\hline & ${ }^{18} \mathrm{~F}-\mathrm{FDM}$ & PET & {$[25]$} \\
\hline \multirow{10}{*}{ Thrombosis } & ${ }^{64} \mathrm{Cu}-\mathrm{FBP} 1,-\mathrm{FBP} 2,-\mathrm{FBP} 3$ & PET/MR & {$[56]$} \\
\hline & ${ }^{64} \mathrm{Cu}-\mathrm{FBP} 7$ & $\mathrm{PET} / \mathrm{CT}$ & {$[33 \bullet \bullet]$} \\
\hline & ${ }^{64} \mathrm{Cu}-\mathrm{FBP} 8$ & $\mathrm{PET} / \mathrm{CT}$ & {$[34]$} \\
\hline & ${ }^{68} \mathrm{Ga}-\mathrm{FBP} 14$ & $\mathrm{PET} / \mathrm{CT}$ & {$[32]$} \\
\hline & ${ }^{111}$ In-FBP15 & $\mathrm{SPECT} / \mathrm{CT}$ & {$[32]$} \\
\hline & ${ }^{99 \mathrm{~m}} \mathrm{Tc}-\mathrm{FBP} 16$ & SPECT/CT & {$[32]$} \\
\hline & ${ }^{111}$ In-FXIII & $\mathrm{SPECT} / \mathrm{CT}$ & {$[35]$} \\
\hline & ${ }^{99 \mathrm{~m}}$ Tc-FXIII & $\mathrm{SPECT} / \mathrm{CT}$ & {$[36]$} \\
\hline & ${ }^{111}$ In-LIBS & $\mathrm{SPECT} / \mathrm{CT}$ & {$[37]$} \\
\hline & ${ }^{18} \mathrm{~F}-\mathrm{scF} v_{\text {anti-LIBS }}$ & $\mathrm{PET} / \mathrm{CT}$ & [38] \\
\hline \multirow[t]{4}{*}{ Plaque angiogenesis } & ${ }^{99 \mathrm{~m}} \mathrm{Tc}-\mathrm{IDA}-\mathrm{D}-[\mathrm{c}(\mathrm{RGDfK})]_{2}$ & $\mathrm{SPECT} / \mathrm{CT}$ & {$[39 \bullet]$} \\
\hline & ${ }^{64} \mathrm{Cu}-\mathrm{NOTA}-3-4 \mathrm{~A}$ & $\mathrm{PET} / \mathrm{CT}$ & {$[40]$} \\
\hline & ${ }^{18} \mathrm{~F}-\mathrm{RGD}-\mathrm{K} 5$ & $\mathrm{PET} / \mathrm{CT}$ & {$[42]$} \\
\hline & ${ }^{99 \mathrm{~m}} \mathrm{Tc}-\mathrm{scVR} 1,{ }^{99 \mathrm{~m}} \mathrm{Tc}-\mathrm{scVR} 2$ & SPECT/CT & {$[43]$} \\
\hline Plaque microcalcification & ${ }^{18} \mathrm{~F}-\mathrm{NaF}$ & $\mathrm{PET} / \mathrm{CT}$ & {$\left[44 \bullet, 46 \bullet, 47^{\bullet}, 48-50\right]$} \\
\hline \multirow[t]{2}{*}{ LDL oxidation } & ${ }^{64} \mathrm{Cu}-3 \mathrm{H} 3-\mathrm{scFv}$ & $\mathrm{PET} / \mathrm{CT}$ & [53] \\
\hline & ${ }^{89} \mathrm{Zr}-\mathrm{LA} 25$ & PET/MR & {$[52]$} \\
\hline \multirow[t]{2}{*}{ Plaque hypoxia } & ${ }^{18} \mathrm{~F}$-FMISO & $\mathrm{PET} / \mathrm{CT}$ & {$[54 \bullet]$} \\
\hline & ${ }^{18} \mathrm{~F}-\mathrm{HX} 4$ & $\mathrm{PET} / \mathrm{CT} / \mathrm{MR}$ & {$[55]$} \\
\hline
\end{tabular}

\section{Emerging Targets for Radionuclide Imaging of Atherothrombosis}

While a variety of radionuclide-based approaches have been applied in the setting of atherosclerosis, novel methods for targeted imaging of vulnerable plaque are continuing to emerge. One recent technique that has recently emerged is molecular imaging of oxidized LDL (oxLDL), which is a hallmark signal representing proinflammatory conditions within plaque. During the process of LDL oxidation, various by-products are generated that have proinflammatory chemical modifications of lipid and protein moieties that are known as oxidation-specific epitopes (OSEs), which could signal dangerously high levels of inflammation in plaque [51]. Therefore, Senders et al. [52] investigated the utility of a novel PET probe, ${ }^{89} \mathrm{Zr}$-LA25, that targeted malondialdehydeacetaldehyde epitopes and found that ${ }^{89} \mathrm{Zr}$-LA25 specifically co-localized to advanced and ruptured atherosclerotic plaques that had accompanying thrombi. Recent work by Sasaki and colleagues [53] has further demonstrated the potential of molecular imaging of oxLDL in atherosclerosis by developing a PET targeted probe, ${ }^{64} \mathrm{Cu}-3 \mathrm{H} 3$-scFv, targeting the B2-glycoprotein/oxLDL complex, with the probe showing colocalization to lipid deposits in atherosclerotic lesions in a rabbit model of hyperlipidemia.
Along with molecular imaging of oxLDL, imaging of hypoxia has also emerged as a potential target for assessing plaque stability. Recent investigations focused on molecular imaging of plaque hypoxia have revealed that uptake of a previously developed radionuclide, ${ }^{18} \mathrm{~F}$-fluoromisonidazole (FMISO), increases in macrophage rich hypoxic regions of plaque in a rabbit model of atherosclerosis [54•]. Additionally, a novel ${ }^{18} \mathrm{~F}$-labeled probe, ${ }^{18} \mathrm{~F}-2-(4-((2-$ nitro- $1 \mathrm{H}$-imidazol-1yl)methyl)-1H-1,2,3-triazol-1-yl)propan-1-ol (HX4), has recently demonstrated potential for the non-invasive detection of plaque hypoxia in patients with carotid artery stenosis, where a combination of PET, CT, and MR images showed that ${ }^{18} \mathrm{~F}-\mathrm{HX} 4$ uptake was specific to regions of atherosclerotic plaque, correlated with ${ }^{18} \mathrm{~F}-\mathrm{FDG}$ uptake (macrophage infiltration) in plaque and correlated with MR-derived measures of carotid artery wall dimensions [55].

\section{Conclusions}

Novel approaches continue to emerge for radionuclide imaging of atherosclerosis and atherothrombosis, with a variety of radiolabeled probes already available in both the pre-clinical and clinical environment for SPECT and PET imaging of vulnerable plaque (Table 1). Although 
numerous physiologic targets have been identified and non-invasively evaluated as potential biomarkers associated with high-risk plaque, the debate continues as to which molecular target provides the most relevant and significant prognostic value for general and clinical populations. Additionally, validation studies that confirm the specificity of radionuclides for their molecular target-of-interest are still warranted before many of these probes can become part of routine clinical use. Regardless of the technique utilized, the high sensitivity imaging characteristics of SPECT and PET should continue to provide unique opportunities for radionuclide-based investigations and offer novel tools for non-invasive detection of, and physiological insight into, atherosclerosis.

\section{Compliance with Ethical Standards}

Conflict of Interest Dr. Stacy has no conflict of interest to declare for this work.

Human and Animal Rights This article does not contain any studies with human or animal subjects performed by the author.

Open Access This article is distributed under the terms of the Creative Commons Attribution 4.0 International License (http:// creativecommons.org/licenses/by/4.0/), which permits unrestricted use, distribution, and reproduction in any medium, provided you give appropriate credit to the original author(s) and the source, provide a link to the Creative Commons license, and indicate if changes were made.

\section{References}

Papers of particular interest, published recently, have been highlighted as:

- Of importance

-• Of major importance

1. Roth GA, Dwyer-Lindgren L, Bertozzi-Villa A, Stubbs RW, Morozoff C, Naghavi M, et al. Trends and patterns of geographic variation in cardiovascular mortality among US counties, 19802014. JAMA. 2017;317:1976-92.

2. Hansson GK. Inflammation, atherosclerosis, and coronary artery disease. N Engl J Med. 2005;352:1685-95.

3. Virmani R, Kolodgie FD, Burke AP, Finn AV, Gold HK, Tulenko $\mathrm{TN}$, et al. Atherosclerotic plaque progression and vulnerability to rupture: angiogenesis as a source of intraplaque hemorrhage. Arter Thromb Vasc Biol. 2005;25:2054-61.

4. Libby P, Ridker PM, Hansson GK. Progress and challenges in translating the biology of atherosclerosis. Nature. 2011;473:31725 .

5. Hansson GK, Libby P, Tabas I. Inflammation and plaque vulnerability. J Intern Med. 2015;278:483-93.

6. Cuadrado I, Saura M, Castejon B, Martin AM, Herruzo I, Balatsos $\mathrm{N}$, et al. Preclinical models of atherosclerosis. The future of hybrid
PET/MR technology for the early detection of vulnerable plaque. Expert Rev Mol Med. 2016;18:e6.

7. Krishnan S, Otaki Y, Doris M, Slipczuk L, Arnson Y, Rubeaux M, et al. Molecular imaging of vulnerable coronary plaque: a pathophysiologic perspective. J Nucl Med. 2017;58:359-64.

8. Honda S, Kataoka Y, Kanaya T, Noguchi T, Ogawa H, Yasuda S. Characterization of coronary atherosclerosis by intravascular imaging modalities. Cardiovasc Diagn Ther. 2016;6:368-81.

9. Detrano R, Guerci AD, Carr JJ, Bild DE, Burke G, Folsom AR, et al. Coronary calcium as a predictor of coronary events in four racial or ethnic groups. N Engl J Med. 2008;358:1336-45.

10. Andrews JPM, Fayad ZA, Dweck MR. New methods to image unstable atherosclerotic plaques. Atherosclerosis. 2018;272:118 28.

11. Stacy MR, Sinusas AJ. Emerging imaging modalities in regenerative medicine. Curr Pathobiol Rep. 2015;3:27-36.

12. Zhang J, Maniawski P, Knopp M. Performance evaluation of the next generation solid-state digital photon counting PET/CT system. EJNMMI Res. 2018;8:97.

13. Caobelli F, Bengel FM. In vivo evaluation of atherosclerotic plaques and culprit lesions using noninvasive techniques. Nat Rev Cardiol. 2015;12:79.

14. Evans NR, Tarkin JM, Chowdhury MM, Warburton EA, Rudd JHF. PET imaging of atherosclerotic disease: advancing plaque assessment from anatomy to pathophysiology. Curr Atheroscler Rep. 2016;18:30.

15. Wang Z, Peter K. Molecular imaging of atherothrombotic diseases. Seeing is believing. Arterioscler Thromb Vasc Biol. 2017;37:1029 40.

16.• Tawakol A, Fayad ZA, Mogg R, Alon A, Klimas MT, Dansky H, et al. Intensification of statin therapy results in a rapid reduction in atherosclerotic inflammation. Results of a multicenter fluorodeoxyglucose-positron emission tomography/computed tomography feasibility study. J Am Coll Cardiol. 2013;62:909-17 This study demonstrated that ${ }^{18}$ F-FDG PET/CT imaging was capable of detecting the therapeutic response to statin therapy in patients with atherosclerosis.

17. Figueroa AL, Abdelbaky A, Truong QA, Corsini E, MacNabb MH, Lavender ZR, et al. Measurement of arterial activity on routine FDG PET/CT images improves prediction of risk of future CV events. JACC Cardiovasc Imaging. 2013;6:1250-9.

18. Duivenvoorden R, Mani V, Woodward M, Kallend D, Suchankova $\mathrm{G}$, Fuster V, et al. Relationship of serum inflammatory biomarkers with plaque inflammation assessed by FDG PET/CT. The dalPLAQUE study. JACC Cardiovasc Imaging. 2013;6:1087-94.

19. Emami H, Vucic E, Subramanian S, Abdelbaky A, Fayad ZA, Du S, et al. The effect of BMS-582949, a P38 mitogen-activated protein kinase (P38 MAPK) inhibitor on arterial inflammation: a multicenter FDG-PET trial. Atherosclerosis. 2015;240:490-6.

20. van der Valk FM, Verweij SL, Zwinderman KAH, Strang AC, Kaiser Y, Marquering HA, et al. Thresholds for arterial wall inflammation quantified by $18 \mathrm{~F}-\mathrm{FDG}$ PET imaging. JACC Cardiovasc Imaging. 2016;9:1198-207.

21. Hellberg S, Sippola S, Liljenback H, Virta J, Silvola JMU, Stahle $\mathrm{M}$, et al. Effects of atorvastatin and diet interventions on atherosclerotic plaque inflammation and $\left.{ }^{18} \mathrm{~F}\right]$ FDG uptake in $\mathrm{Ldlr}^{-1}$ Apob $^{100 / 100}$ mice. Atherosclerosis. 2017;263:369-76.

22. Iwatsuka R, Matsue Y, Yonetsu T, O'uchi T, Matsumura A, Hashimoto Y, et al. Arterial inflammation measured by $18 \mathrm{~F}-\mathrm{FDG}-$ PET-CT to predict coronary events in older subjects. Atherosclerosis. 2018;268:49-54.

23. van der Valk FM, Bekkering S, Kroon J, Yeang C, den Bossche JV, van Buul JD, et al. Oxidized phospholipids on lipoprotein(a) elicit arterial wall inflammation and an inflammatory monocyte response in humans. Circulation. 2016;134:611-24. 
24. Cocker MS, Spence JD, Hammond R, DeKemp RA, Lum C, Wells $\mathrm{G}$, et al. [18F]-fluorodeoxyglucose PET/CT imaging as a marker of carotid plaque inflammation: comparison to immunohistology and relationship to acuity of events. Int J Cardiol. 2018;271:378-86.

25. Tahara N, Mukherjee J, de Haas HJ, Petrov AD, Tawakol A, Haider $\mathrm{N}$, et al. 2-deoxy-2-[18F]fluoro-D-mannose positron emission tomography imaging in atherosclerosis. Nat Med. 2014;20:215-9.

26. Tarkin JM, Joshi FR, Evans NR, Chowdhury MM, Figg NL, Shah $\mathrm{AV}$, et al. Detection of atherosclerotic inflammation by $68 \mathrm{Ga}-$ DOTATATE PET compared to [18F] FDG PET imaging. J Am Coll Cardiol. 2017;69:1774-91 This study demonstrated that ${ }^{68}$ Ga-DOTATATE PET/CT imaging allows for improved identification of vulnerable coronary plaque beyond the traditional approach of ${ }^{18}$ F-FDG imaging.

27. Majmudar MD, Yoo J, Keliher EJ, Truelove JJ, Iwamoto Y, Sena B, et al. Polymeric nanoparticle PET/MR imaging allows macrophage detection in atherosclerotic plaques. Circ Res. 2013;122:755-61.

28. Li X, Bauer W, Israel I, Kreissl MC, Weirather J, Richter D, et al. Targeting P-selectin by gallium-68-labeled fucoidan positron emission tomography for noninvasive characterization of vulnerable plaques. Correlation with in vivo 17.6T MRI. Arterioscler Thromb Vasc Biol. 2014;34:1661-7.

29. Liu C, Zhang X, Song Y, Wang Y, Zhang F, Zhang Y, et al. SPECT and fluorescence imaging of vulnerable atherosclerotic plaque with a vascular cell adhesion molecule 1 single-chain antibody fragment. Atherosclerosis. 2016;254:263-70.

30. Bala G, Blykers A, Xavier C, Descamps B, Broisat A, Ghezzi C, et al. Targeting of vascular cell adhesion molecule-1 by $18 \mathrm{~F}$ labelled nanobodies for PET/CT imaging of inflamed atherosclerotic plaques. Eur Hear J Cardiovasc Imaging. 2016;17:1001-8.

31. Weiberg D, Thackeray JT, Daum G, Sohns JM, Kropf S, Wester H$\mathrm{J}$, et al. Clinical molecular imaging of chemokine receptor CXCR4 expression in atherosclerotic plaque using 68Ga-pentixafor PET: correlation with cardiovascular risk factors and calcified plaque burden. J Nucl Med. 2018;59:266-72.

32. Oliveira BL, Blasi F, Rietz TA, Rotile NJ, Day H, Caravan P. Multimodal molecular imaging reveals high target uptake and specificity of $111 \mathrm{In}$ - and $68 \mathrm{Ga}$-labeled fibrin-binding probes for thrombus detection in rats. J Nucl Med. 2015;56:1587-92.

33.• Ay I, Blasi F, Rietz TA, Rotile NJ, Kura S, Brownell AL, et al. In vivo molecular imaging of thrombosis and thrombolysis using a fibrin-binding positron emission tomographic probe. Circ Cardiovasc Imaging. 2014;7:697-705. This study developed and applied a novel fibrin targeted probe for evaluating a rat model of thrombosis and demonstrated the ability of PET/CT imaging to detect the response to thrombolysis treatment.

34. Blasi F, Oliveira BL, Rietz TA, Rotile NJ, Naha PC, Cormode DP, et al. Multisite thrombus imaging and fibrin content estimation with a single whole-body PET scan in rats. Arterioscler Thromb Vasc Biol. 2015;35:2114-21.

35. Chen JW, Figueiredo J-L, Wojtkiewicz GR, Siegel C, Iwamoto Y, Kim D-E, et al. Selective factor XIIa inhibition attenuates silent brain ischemia. Application of molecular imaging targeting coagulation pathway. JACC Cardiovasc Imaging. 2012;5:1127-38.

36. Zhuang ZW, Huang Y, Ju R, Maxfield MW, Ren Y, Wang X, et al. Molecular imaging of factor XIII activity for the early detection of mouse coronary microvascular disease. Theranostics. 2019;9: 1474-89.

37. Heidt T, Deininger F, Peter K, Goldschmidt J, Pethe A, Hagemeyer $\mathrm{CE}$, et al. Activated platelets in carotid artery thrombosis in mice can be selectively targeted with a radiolabeled single-chain antibody. PLoS One. 2011;6:e18446.

38. Ardipradja K, Yeoh SD, Alt K, O'Keefe G, Rigopoulos A, Howells DW, et al. Detection of activated platelets in a mouse model of carotid artery thrombosis with $18 \mathrm{~F}$-labeled single-chain antibodies. Nucl Med Biol. 2014;41:229-37.
39. Yoo JS, Lee J, Jung JH, Moon BS, Kim S, Lee BC, et al. SPECT/ CT imaging of high-risk atherosclerotic plaques using integrinbinding RGD dimer peptides. Sci Rep. 2015;5:11752 This study evaluated a novel $\alpha v \beta 3$ integrin targeted probe for SPECT/CT imaging and validated the findings from imaging studies with autoradiography and histology.

40. Jiang L, Tu Y, Kimura RH, Habte F, Chen H, Cheng K, et al. 64Culabeled divalent cystine knot peptide for imaging carotid atherosclerotic plaques. J Nucl Med. 2015;56:939-44.

41. Parma L, Baganha F, Quax PHA, de Vries MR. Plaque angiogenesis and intraplaque hemorrhage in atherosclerosis. Eur J Pharmacol. 2017;816:107-15.

42. Golestani R, Mirfeizi L, Zeebregts CJ, Westra J, de Haas HJ, Glaudemans AWJM, et al. Feasibility of [18F]-RGD for ex vivo imaging of atherosclerosis in detection of avB3 integrin expression. J Nucl Cardiol. 2015;22:1179-86.

43. Tekabe Y, Johnson LL, Rodriquez K, Li Q, Backer M, Backer JM. Selective imaging of vascular endothelial growth factor receptor-1 and receptor- 2 in atherosclerotic lesions in diabetic and nondiabetic ApoE-/- mice. Mol Imaging Biol. 2018;20:85-93.

44.• Irkle A, Vesey AT, Lewis DY, Skepper JN, JLE B, Dweck MR, et al. Identifying active vascular microcalcification by $18 \mathrm{~F}$ sodium fluoride positron emission tomography. Nat Commun. 2015;6:7495 This study demonstrated that ${ }^{18}$ F-NaF can differentiate between macro- and microcalcification in plaque.

45. Blau M, Ganatra R, Bender MA. 18F-fluoride for bone imaging. Semin Nucl Med. 1972;2:31-7.

46. Derlin T, Wisotzki C, Richter U, Apostolova I, Bannas P, Weber C, et al. In vivo imaging of mineral deposition in carotid plaque using $18 \mathrm{~F}$-sodium fluoride PET/CT: correlation with atherogenic risk factors. J Nucl Med. 2011;52:362-8. This study demonstrated the ability of ${ }^{18} \mathrm{~F}-\mathrm{NaF}$ to detect calcified plaque and showed a correlation between ${ }^{18} \mathrm{~F}$-NaF uptake and multiple cardiovascular risk factors.

47. Kitagawa T, Yamamoto H, Toshimitsu S, Sasaki K, Senoo A, Kubo $\mathrm{Y}$, et al. 18F-sodium fluoride positron emission tomography for molecular imaging of coronary atherosclerosis based on computed tomography analysis. Atherosclerosis. 2017;263:385-92.

48. Dweck MR, Chow MWL, Joshi NV, Williams MC, Jones C, Fletcher AM, et al. Coronary arterial $18 \mathrm{~F}$-sodium fluoride uptake. A novel marker of plaque biology. J Am Coll Cardiol. 2012;59: 1539-48.

49. Joshi NV, Vesey AT, Williams MC, Shah ASV, Calvert PA, Craighead FHM, et al. 18F-fluoride positron emission tomography for identification of ruptured and high-risk coronary atherosclerotic plaques: a prospective clinical trial. Lancet. 2014;383:705-13.

50. Cocker MS, Spence JD, Hammond R, Wells G, DeKemp RA, Lum $\mathrm{C}$, et al. [18F]-NaF PET/CT identifies active calcification in carotid plaque. JACC Cardiovasc Imaging. 2017;10:486-8.

51. Binder CJ, Papac-Milicevic N, Witztum JL. Innate sensing of oxidation-specific epitopes in health and disease. Nat Rev Immunol. 2016;16:485-97.

52. Senders ML, Que X, Cho YS, Yeang C, Groenen H, Fay F, et al. PET/MR imaging of malondialdehyde-acetaldehyde epitopes with a human antibody detects clinically relevant atherothrombosis. J Am Coll Cardiol. 2018;71:321-35.

53. Sasaki T, Kobayashi K, Kita S, Kojima K, Hirano H, Shen L, et al. In vivo distribution of single chain variable fragment $(\mathrm{scFv})$ against atherothrombotic oxidized LDL/B2-glycoprotein I complexes into atherosclerotic plaques of WHHL rabbits: implications for clinical PET imaging. Autoimmun Rev. 2017;16:159-67.

54. Mateo J, Izquierdo-Garcia D, Badimon JJ, Fayad ZA, Fuster V. Noninvasive assessment of hypoxia in rabbit advanced atherosclerosis using $18 \mathrm{~F}$-fluoromisonidazole positron emission tomographic imaging. Circ Cardiovasc Imaging. 2014;7:312-20 This study was 
one of the first to demonstrate the feasibility of hypoxia targeted imaging in atherosclerotic plaque.

55. van der Valk FM, Sluimer JC, Voo SA, Verberne HJ, Nederveen AJ, Windhorst $\mathrm{AD}$, et al. In vivo imaging of hypoxia in atherosclerotic plaques in humans. JACC Cardiovasc Imaging. 2015;8:1340-1.
56. Ciesienski KL, Yang Y, Ay I, Chonde DB, Loving GS, Rietz TA, et al. Fibrin-targeted PET probes for the detection of thrombi. Mol Pharm. 2013;10:1100-10.

Publisher's Note Springer Nature remains neutral with regard to jurisdictional claims in published maps and institutional affiliations. 